\title{
El colonialismo y la colonialidad curricular en los Estudios Generales. Apuntes para una discusión ${ }^{1}$
}

\section{Colonialism and curricular coloniality in General Education. Notes for a discussion}

Recibido: 3 de octubre de 2021 | Aprobado: 23 de noviembre de 2021

\section{Resumen}

Este artículo visita el origen y desarrollo de la Facultad de Estudios Generales de la Universidad de Puerto Rico que integró, en su sociogénesis, algunas variantes del pensamiento orteguiano y que desembocó en las visiones del movimiento denominado "General Education" impulsado canónicamente desde fulcros de poder intelectual nordeurocéntricos e impuesto a la realidad puertorriqueña con significativas consecuencias. Como objetivo, nos interesa examinar los supuestos de filosofía educativa y de currículo que prevalecieron para detectar en ellos un importante y determinante episodio de colonialismo intelectual. Con el lente de la decolonialidad y la crítica a la triple colonialidad- del ser, el saber y el poder- misma que impregnó el programa académico de umbral de los estudiantes universitarios de la UPR en Río Piedras, intentamos una exposición de las razones por las que la academia puertorriqueña se subsumió en un caldo eurocéntrico que retrasó su sociogénesis identitaria. La metodología elegida consiste en el análisis de contenido de documentos que recogen la fundación de las principales instituciones académicas y estrategias curriculares y del proceso de difusión intensiva del "General Education Movement" en la Facultad de Estudios Generales. Identificamos como vetas plausibles para el análisis ulterior cursos, seminarios, actividades y programas. Como sugerencia de cierre, consideramos que nuestro compromiso es aportar para que el canon occidental se entienda como una forma, válida también, entre otras, de acercarnos al conocimiento y a la educación, pero de ninguna manera excluyente. Este trabajo tiene por pivote la crítica al 'sistema mundo' y los valores hegemónicos de la modernidad que le son inherentes, con la propuesta de la decolonialidad como lente crítico emplazador de un acontecer educativo que se articula con criterios avasalladores de dominación epistémica.

Palabras clave: Currículo, Estudios Generales, Facultad de Estudios, Universidad de Puerto Rico; Colonialidad del saber; Colonialismo

1 Artículo derivado de la ponencia presentada en junio de 2021 en el XII Simposio Internacional de Estudios Generales (modalidad virtual). Pontificia Universidad Católica Madre y Maestra (PUCMM), República Dominicana, y Red Internacional de Estudios Generales (RIDEG).

* Doctor y catedrático en la Universidad de Puerto Rico. Decano de la Facultad de Estudios Generales. Para contactar al autor: carlos.sanchez8@upr.edu

ISSN (en línea): 1814-4152 / Sitio web: http://cuaderno.pucmm.edu.do

CÓMO CITAR: Sánchez Zambrana, C. (2022). El colonialismo y la colonialidad curricular en los Estudios Generales. Apuntes para una discusión. Cuaderno de Pedagogía Universitaria, 19 (37), 47-59. 


\section{Abstract}

This article visits the origin and development of the Faculty of General Education of the University of Puerto Rico that integrated, in its sociogenesis, some variants of Ortega's thought and that led to the visions of the movement called "General Education" canonically promoted from fulcrums of power. North-European intellectual and imposed on the Puerto Rican reality with significant consequences. As an objective, we are interested in examining the assumptions of educational philosophy and of the curriculum that prevailed to detect in them an important and decisive episode of intellectual colonialism. With the lens of decoloniality and the critique of the triple coloniality - of being, knowledge and power - itself that permeated the threshold academic program of the university students of the UPR in Río Piedras, the author attempts an exposition of the reasons for which the Puerto Rican academy subsumed itself in a Eurocentric soup that delayed its identity sociogenesis. The chosen methodology consists of the content analysis of documents that collect the foundation of the main academic institutions and curricular strategies and of the intensive dissemination process of the "General Education Movement" in the Faculty of General Education. We identify as plausible veins for further analysis courses, seminars, activities and programs. As a closing suggestion, we consider that our commitment is to contribute so that the Western canon is understood as a valid way, among others, of approaching knowledge and education, but in no way exclusive. The pivot of this work is the critique of the 'world system' and the hegemonic values of modernity that are inherent in it, with the proposal of decoloniality as a critical lens that sets off an educational event that is articulated with overwhelming criteria of epistemic domination.

Keywords: Curriculum, General Education, Universidad de Puerto Rico; Coloniality of knowledge, Colonialism

\section{Introducción}

Ni redonda ni lineal, la 'policrisis' estalló. Y con ella se abre todo un campo para reflexionar sobre el contexto actual en la educación superior y, en consecuencia, sobre la pertinencia de atender las necesidades académicas (estudiantiles, docentes, institucionales y comunitarias) urgidas de esta policrisis realmente existente. Se habla de policrisis para destacar las múltiples facetas de estos resquebrajamientos estructurales y complejos. Azócar (2012), entre otros, desarrolla el concepto de Edgard Morin:

La crisis ecológica se acentúa con la degradación creciente de la biosfera (lo que provocará nuevas crisis económicas, sociales y políticas) ...La crisis de las sociedades tradicionales deriva de la occidentalización que tiende a desintegrarlas....La civilización occidental, que produce las crisis de la globalización, está ella misma en crisis. Los efectos egoístas del individualismo destruyen la antigua solidaridad... La crisis de la modernidad occidental ocasiona que las modernas soluciones para la crisis sean irrisorias... La crisis demográfica se amplifica a causa de la conjunción de diferentes fenómenos: la superpoblación de los países pobres, la disminución de la población de la mayoría de los países ricos y el desarrollo de los flujos migratorios engendra esa miseria...La crisis del mundo rural es una crisis de desertificación, provocada por la importante concentración urbana y la extensión de los monocultivos industrializados, entregados a los pesticidas, privados de vida animal, así como por las dimensiones de la ganadería industrializada, productora de alimentos degradados de las hormonas y los antibióticos...

Y nosotros agregamos con doliente severidad, precipitada por la 'Pandemia' tremebunda del 2020, que se revela al modo de fase catastrófica del sistema-mundo y la modernidad.

Hagamos un poco de historia provincial. En la Universidad de Puerto Rico nos es grato y frecuente escuchar de parte de exalumnos y la comunidad referidos de elogio hacia los cursos de primer año. ¡Qué importantes fueron esos cursos! - exclaman. 
De contramano hay que apresurarse a indicar que también es constatable la voracidad de las facultades especializadas por colonizar la malla curricular de esas experiencias académicas de primer año focalizadas en la Facultad de Estudios Generales. Y esto es así desde el 'Plan Bueso' en los cincuenta hasta la reconceptualización del Bachillerato Universitario en $2006{ }^{1}$

En un balance, la estimativa sobre los llamados cursos básicos, sobre la experiencia de inicio y del marullo de emociones sentipensante ${ }^{2}$ alrededor de este umbral son de muy alta justipreciación. No lo digo únicamente como una expresión testimonial derivada de una historia de vida que surca ya sus cuatro décadas, sino- y es lo relevante- como parte de un conglomerado tupido y enmarañado de mitificaciones que tiene y contiene, que porta, exporta y aporta la denominada Educación General en Puerto Rico, y de los que me he venido ocupando este último lustro, junto a un tinglado de colegas invaluables. Si esta valoración de excelencia ha permeado en el imaginario de generaciones que han pasado por sus selvas epistémicas y volitivas, entiéndase el salón de clases, profiriendo y exaltando esta proficiencia, vale bien una alerta desde 'el más acá' para advertir que, al así hacerlo, nos hemos convertido en cómplices y voceros de uno de los episodios más intensos y penetrantes de colonialidad curricular. En nuestro país, sin duda, y posiblemente válido para el Caribe.

Este discurso tiene por pivote la crítica al 'sistema mundo', y los valores hegemónicos de la modernidad que le son inherentes, con la propuesta de la decolonialidad como lente crítico emplazador de un acontecer educativo que se articula con esos criterios avasalladores de dominación epistémica. Se visita el origen y desarrollo de la Facultad de Estudios Generales de la Universidad de Puerto Rico que integró, en su sociogénesis, algunas variantes del pensamiento orteguiano y que desembocó en las visiones del movimiento denominado "General Education" impulsado canónicamente desde fulcros de poder intelectual nordeurocéntricos y adaptado a la realidad puertorriqueña con significativas consecuencias. Nos interesa examinar los supuestos de filosofía educativa y de currículo que prevalecieron para detectar en ellos un importante y determinante episodio de triple colonialidad- del ser, el saber y el poder- que impregnó el programa académico de umbral de los estudiantes universitarios de la UPR en Río Piedras.

El ensayo aspira a generar una discusión en torno al concepto colonialidad curricular ${ }^{3}$ en la trayectoria histórica de la educación general en Puerto Rico y en la Facultad de Estudios Generales (FEG), principal unidad académica encargada de impartirle. Se trata de apenas un bosquejo de ideas en desarrollo. ¿Es posible diferenciar una praxis de colonialidad más allá del colonialismo educativo que es presumible exista en toda colonia, aún en una tan compleja como la que se ha producido por siglos y aún se reproduce sobre la nación puertorriqueña?

Como metodología, trabajamos con fuentes primarias de la época fundacional y de la década de los cincuenta (informes anuales, prensa estudiantil, actas, minutas de los organismos académicos de jerarquía) que representan el color del momento en donde se fundaron las principales instituciones académicas y estrategias curriculares

1 Ver Sánchez, Vélez, Maldonado; (2013) La Educación General en Puerto Rico: la década de los cincuenta; Ediciones Mágica, Río Piedras. El 'Plan Bueso' consistía en sustraer- para los estudiantes con vocación hacia la ciencia- los créditos de las ciencias naturales en educación general y llevarlos directamente a las materias especializadas en la Facultad de Ciencias Naturales. Y en la certificación \# 46 se oficializa un cercenamiento de los créditos del componente de Educación General bajo el palio y amparo de la Facultad de Estudios Generales.

2 Nos dice Deyby Rodrigo Espinosa Gómez en su escrito Una educación sentipensante: hacia una escuela diferente pronunciado en el $1^{\circ}$ Concurso de Artículos Grupo Geard: El concepto sentipensante nace de aquellas sabias palabras de los pescadores en San Benito Abad (Sucre) al sociólogo Orlando Fals Borda: "Nosotros actuamos con el corazón, pero también empleamos la cabeza, y cuando combinamos las dos cosas así, somos sentipensantes", un concepto que ha inspirado a poetas, tal fue el caso de Eduardo Galeano quien le definió como "aquel lenguaje que dice la verdad". En el campo educativo, significa "aprender a sentir y pensar al otro" (Espinosa, 2014), ser sujetos de praxis (Ghiso, 2013). Es reconocer la escuela como un espacio emocional, vivo, donde circula un universo de diferencias pensantes, comprende la educación no para el mundo de las competencias, sino para el reconocimiento y defensa de la dignidad humana.

3 Empleamos el concepto en el sentido en que lo ha realizado Sebastian Rinaldi en su aproximación a una decolonialidad de la ciencia política. Ver: Sebastián Rinaldi (2019b): "Perspectiva nordeurocéntrica en el currículum universitario de ciencia política", Revista Contribuciones a las Ciencias Sociales, (marzo 2019). En línea: https://www.eumed.net/rev/cccss/2019/03/curriculum-ciencia-politica.html //hdl.handle.net/20.500.11763/cccss1903curriculum-ciencia-politica 
y del proceso de difusión intensiva del "General Education Movement" en la FEG. A lo largo del texto, examinaremos cursos, seminarios, docentes (profesores invitados) y programas (el Programa de Bachillerato en Artes en Educación General), así como el entretejido de actividades co-curriculares que se gestaron, (viajes académicos, el militarismo estudiantil, el papel de las fundaciones, todos con un marcado énfasis en el nordeúrocentrismo y en el privilegio de los valores "universales" de la cultura occidental. Finalmente, ofreceremos las conclusiones a las que arriba esta reflexión.

\section{Apuntes metodológicos}

¿Quién sabe si fue de Julio Cortázar, en su Discurso del no método, método del no discurso, y así vamos, una suerte de pionero? $\mathrm{O}$, tal vez, proviene de cuños de más reciente inspiración a partir de las inquietudes de Walsh et al. (2002). O con el acicate de Walter Mignolo (2010), quien promulga una acción de Desobediencia epistémica ${ }^{4}$. Lo cierto es que el asunto de la decolonialidad en la metodología ha comenzado a ser abordado frontalmente. Identifico, además, en este afán al chileno Alejandro Haber quien impulsara su indisciplina metodológica y su concepto de nometodología y a María Eugenia Borsani cuando propone sus Reconstrucciones Metodológicas y/o Metodologías a Posteriori (2014).

Invito a su lectura pues contiene, para el terreno en que nos desenvolvemos cotidianamente, vale decir, en esta selva llamada academia, valiosas y muy sinceras pistas de sobrevivencia. Sostiene Borsani (2014):

Lo problemático estriba en su condición de colonizada, lo que es lo mismo decir que es el resultado de una imposición colonial en conformidad con los patrones de conocimiento euro-centrados. Dicha imposición responde a una estandarización del saber con pretensión de universalidad y neutralidad, según un reducido universo de problemas que es el que las ciencias sociales y las humanidades modeladas según lo que los patrones occidentales, reconocen como tales. Por ello, nuestra inquietud, disgusto o desasosiego no se sostiene sino en la dimensión colonial de la metodología, en advertir que es un área más de las tantas en las que ha hecho lo suyo la colonialidad del saber y sigue haciéndolo. (p. 152).

Por su parte, Haber (2011) ya había resumido:

Si nos preguntásemos por la posibilidad de una investigación decolonial, habría que pensar en la aptitud de la metodología de investigación para tal propósito, y si en todo caso la razón decolonial no habría de ocuparse de decolonizar la metodología. En este trabajo me propongo aportar a la reflexión metodológica en clave decolonial, que encuentro seriamente vacante. Partiendo de la idea de la inmediatez vestigial perdida en la investigación moderna, y ligándola con la cisura y la ruptura operadas por la razón moderna colonial, propongo un marco nometodológico que desplaza la investigación desde la objetualización hacia la situacionalidad. La nometodología adopta la forma de una arqueología indisciplinada, una conversación ampliada en la inmediatez de espaciotiempos discontinuados en la colonialidad. Centrando la táctica en la conversación, la relaciono con la situación de investigación, la escritura, y la mudanza pos occidental de su domicilio hacia el país de los bárbaros. (p. 9).

Desde nuestro enfoque, deseamos aportar lo siguiente. Tres vías, cuando menos, nutren nuestro esfuerzo por caminar el cauce de esta investigación: la nutriente foucaultiana, la nutriente transdisciplinar y la vía decolonial. Para este ensayo, me concentraré en la última. En efecto, es en la tercera vía de este entramado metodológico donde nos insertaremos desde una perspectiva decolonial con la esperanza de poder enriquecer el diálogo con respecto a los múltiples y sutiles modos de expoliación que ha sufrido Puerto Rico por vía de la ‘mala educación' y sus demonios. Y de ahí partiremos para propuestas educativas auténticas y comprometidas con las soluciones apremiantes que presenta una sociedad en policrisis.

4 Borsani le interpreta en el sentido de que Mignolo concibe "un cambio en el posicionamiento respecto al saber y al mundo, dicho de otro modo, un viraje (vuelco) respecto a qué cosas importan hoy, que coadyuven en la construcción de una razón decolonial, para lo cual es imprescindible una torsión respecto a la concepción de razón y de racionalidad de cuño moderno, en pos de ir ligando, vinculando globalmente diversos empeños descolonizantes que se desprendan, es decir se desvinculen críticamente del horizonte imperial”. Op cit .p 156. 
Decolonialidad dialoga con su antónimo. Y colonialidad no significa lo mismo que colonialismo. Señala, por su parte, Nelson Maldonado Torres (2007):

Colonialismo denota una relación jurídicopolítica y económica, en la cual la soberanía de un pueblo reside en el poder de otro pueblo o nación, lo que constituye a tal nación en un imperio. Distinto de esta idea, la colonialidad se refiere a un patrón de poder que emergió como resultado del colonialismo moderno, pero que, en vez de estar limitado a una relación formal de poder entre dos pueblos o naciones, más bien se refiere a la forma como el trabajo, el conocimiento, la autoridad y las relaciones intersubjetivas se articulan entre sí, a través del mercado capitalista mundial y de la idea de raza. Así, pues, aunque el colonialismo precede a la colonialidad, la colonialidad sobrevive al colonialismo. La misma se mantiene viva en manuales de aprendizaje, en el criterio para el buen trabajo académico, en la cultura, el sentido común, en la autoimagen de los pueblos, en las aspiraciones de los sujetos, y en tantos otros aspectos de nuestra experiencia moderna. En un sentido, respiramos la colonialidad en la modernidad cotidianamente. (p.131).

Con este norte, nos encontramos en la exploración de la veta en varias áreas de conocimiento y estuarios cognitivos. Coincidimos, por ejemplo, con Rinaldi (2019a) en las formas en que ha emplazado el asunto en el terreno disciplinar de la más antigua de las ciencias sociales, la política. ${ }^{5}$ Le cito inextenso:

El trabajo nos permite concluir en que la colonialidad aún atraviesa profundamente el currículum de la carrera en sus diferentes etapas, planteándose la posibilidad de repensar los procesos de planificación y enseñanza desde una perspectiva crítica latinoamericana... En el presente trabajo se realizó una descripción de los aspectos que dan cuenta de la presencia de una matriz nordeurocéntrica en las diferentes instancias curriculares de las licenciaturas en Ciencia Política, proponiendo una salida de este esquema de enseñanza y planificación educativa a través de una perspectiva crítica latinoamericana....Tal como se expuso, desde los inicios de nuestros sistemas universitarios se consagró una forma de ver el mundo, y por ende, de hacer ciencias sociales, que se centró en el estudio y la reproducción de las grandes teorías nordeuropeas, cuestión que sigue vigente mayoritariamente en las aulas y sobre la cual se enfoca mayormente el proceso de formación...Hoy más que nunca la ciencia política necesita una mirada distinta a la hora de encarar el diseño de planes y programas analíticos. El desafío que enfrentamos es el de promover investigaciones que tengan una mirada crítica sobre el currículo y las estructuras de enseñanza que persisten otorgando preeminencia a determinadas corrientes de pensamiento que invisibilizan y minimizan las producciones consideradas "alternativas" o "periféricas". Para ello se requiere repensar las categorías desde las cuales se gesta la formación universitaria, pero a la vez rediseñar el sistema científico-académico que permite el desarrollo profesional de los politólogos. Al asumir la enseñanza en la disciplina desde una perspectiva crítica latinoamericana se potencian las posibilidades de conducir la educación hacia un proceso de decolonialidad que culmine con la visión de que existe una única y adecuada manera de preparar a los estudiantes para su ejercicio laboral. En definitiva, de la desobediencia epistemológica puede nacer una nueva y mejor ciencia política que fomente la participación de los expertos comprendiendo las singularidades del contexto de actuación y siendo su ejercicio significativo para la vida política de sus comunidades.

Veamos otro caso importante desde otra pradera epistémica: Ios denominados Estudios

6 Los 'Estudios Latinoamericanos' tienen en la UNAM una historia muy interesante. El 13 de enero de 1960 el Honorable Consejo Técnico de la entonces Escuela Nacional de Ciencias Políticas y Sociales aprobó el plan de estudios y el reglamento interno del Centro de Estudios Latinoamericanos. El Dr. Pablo González Casanova y el Prof. Ezequiel Martínez Estrada pronunciaron los discursos inaugurales el 1 de febrero de 1960. A partir de 1973, con la creación del Posgrado en Estudios Latinoamericanos en la Facultad de Ciencias Políticas y Sociales, los investigadores se incorporaron a la planta docente de los programas de Maestría y Doctorado de la especialidad. De 1992 hasta 1999, el CELA participó en la División de Estudios de Posgrado (DEP) de la Facultad de Ciencias Políticas y Sociales (FCPyS) coordinando dicho Programa. Entre 1995 y 1998, participó activamente en las discusiones sobre los planes de estudios que dieron origen al actual Programa de Posgrado en Estudios Latinoamericanos de la UNAM, integrado por cinco entidades académicas de la Universidad, entre las que se encuentra la Facultad de Ciencias Políticas y Sociales. Tomado de politocascas.unam.mx 
Latinoamericanos y, en especial, la experiencia en la Universidad Nacional Autónoma de México (UNAM), principalísimo centro de difusión de ideas en tal "especialidad" no especializada. ${ }^{6}$

Desde mediados del siglo pasado constituyen un programa de posgrado que ofrece maestría, doctorado y plataformas de investigación/ divulgación sobre la región latinoamericana. ${ }^{7}$

Fundados en los años sesenta con cátedras del ex Rector de la UNAM, Don Pablo González Casanova, reciben influencias muy notables de las emigraciones intelectuales que lograban escapar de las garras de los gobiernos militares de la región ${ }^{8}$. A la par, gestaba sus propios derroteros cobijados por el prestigioso Centro de Estudios Latinoamericanos (CELA). Muchas de estas líneas de investigación emergen vinculadas a una "perspectiva periférica, anticolonialista y antiimperialista que se ha elaborado sobre el principio común de diferenciación respecto al conocimiento producido en los grandes centros de poder" (Sosa Elízaga, 2005, pp. 30-148). ${ }^{9}$

De manera palmaria, el desarrollo curricular de los Estudios Latinoamericanos se iba a producir en un contexto de arduas luchas paradigmáticas. Resultará inevitable, para una búsqueda ontogenética e identitaria de unos estudios latinoamericanos auténticos y robustos, resistir a las ráfagas de colonialidad que se ejercen desde los centros de poder y de saber. Así lo formula Sosa Elízaga (2005) cuando caracteriza y denuncia el problema como uno inserto en -y víctima a la vez- de:

Las continuas presiones extraacadémicas por la adopción de definiciones ideológicas y conceptuales rígidas, usualmente asociadas al financiamiento de la investigación y el carácter frecuentemente subordinado de intelectuales y académicos a la investigación social que se desarrolla en Europa y los Estados Unidos de
América, por razones de estatus, protagonismo y búsqueda de trascendencia, ajenas a las búsquedas o necesidades de conocimiento de sus propias sociedades. (p. 132).

En los documentos constitutivos actuales de la UNAM se desprende la concepción de los Estudios Latinoamericanos, desde una "revisión crítica de los límites de la estrategia de desarrollo postulada por la CEPAL en los años cincuenta y la ruptura con los paradigmas de la sociología norteamericana predominante durante los años sesenta en las ciencias sociales latinoamericanas". (Plan de Estudios de Programa de Posgrado en Estudios Latinoamericanos Gaceta UNAM, 2018). Los límites del cuadrilátero conceptual han estado muy definidos por el canon. De haber lucha contra el canon, esta se daría dentro del canon ${ }^{10}$. Se privilegia el tratamiento de temas teóricos, hijos todos de la adscripción mimética o, tal vez, reciclada de paradigmas euro-centrípetos como la infinita discusión sobre los modos de producción y los debates en torno a los procesos de modernización, v.g.

Sin embargo, concurro plenamente con Sosa Elízaga (2005) que la tarea, en lo formal metodológico, no es solo de mera reconstrucción estadística, sino de replanteamiento general de las propias fuentes de información. Lo cual implica la "construcción de una metodología que nos permita captar el movimiento en el tiempo y en el espacio" e "incluye no solo un trabajo transdisciplinario en la identificación de los territorios involucrados en cada objeto de estudio" (Sosa Elízaga, 2005). Además, involucra cierta intuición poética que solo la pasión transdisciplinaria puede inspirar.

Vivimos en sociedades en que la vida social transcurre a velocidades y con matices de complejidad tal que difícilmente son captadas

7 El autor se recibió de Magister en Estudios Latinoamericanos (Facultad de Ciencias Políticas y Sociales) en enero de 1983 y del doctorado en la misma institución en agosto de 1987

8 Podemos mencionar- sin pretender ser exhaustivos- los nombres de Rodolfo Piuigrós, Sergio Bagú, Adolfo Gilly, Guillermo Almeyra, Carlos Rama, Gerard Pierre-Charles, Susy Castor, Teotonio Dos Santos, Vania Bambirra, Clodomiro Almeyda, Hugo Zemelman, Agustín Cueva, René Zavaleta, José Luis González entre otros.

9 Vid. Sosa R. (2005) Herencias y retos En: Sociologias, Porto Alegre, año 7 no. 14, jul/dic. 2005, pp. 30-148. Su ensayo se dirige a evaluar el peso de las orientaciones dominantes, como las perspectivas de construcción de un pensamiento crítico en la América Latina. Raquel Sosa Elízaga sabe lo que está evaluando. La recuerdo en los años ochenta como adjunta de profesor del importante escritor ecuatoriano Agustín Cueva. Luego, ocupó la Coordinación de la Maestría y Doctorado en Estudios Latinoamericanos de la Facultad de Ciencias Políticas y Sociales.

10 Enuncio canon para las universidades en sentido figurado y siguiendo a C.W. Mills y a V. Pareto, cuando emplean la imagen de élites del poder que se recirculan y retroalimentan. 
por la investigación social canónica ${ }^{11}$. Y si los responsables de elaborar conocimiento se colocan a la zaga de los cambios realmente existentes, mal podríamos posicionarnos en la educación en pro de la transformación social integral.

Observo tangencias en sus ríos de expresión, sus procesos de construcción del conocimiento y de reelaboración permanente de las identidades epistémicas que persiguen. Ello les hermana en problemas, valladares, retos y posibilidades. Coincido con Sosa Elízaga (2005) cuando sostiene que "...replantear, reconstruir los instrumentos y métodos del conocimiento con ese dimensionamiento del tiempo y el espacio, de sujetos y territorios, es, indudablemente, un reto contemporáneo formidable para los Estudios Latinoamericanos". Los reclamos de pertinencia de la educación general, sostenemos por nuestra parte, atraviesan por desafíos similares.

Las posibilidades analíticas de estas convergencias entre los estudios regionales y los estudios generales constituyen un tema aporético en sí mismo; hilos que se desprenden de este texto, pero cuyo tratamiento tengo que dejar en el tintero por el momento. (v.g. el posicionamiento ante las disciplinas: multi, inter o trans; la integración del conocimiento: aditiva o sinérgica; espontánea o por diseño; el sentido y la cualificación sobre las fuentes del saber).

La propia enunciación de 'Latinoamérica' está sobre el tapete de la honestidad académica. Enunciamos Abya Yala ${ }^{12}$ desde la convicción que ello implica por la toma de postura, el decantamiento de nuestros criterios axiológicos y la transparencia en cuanto al poliálogo ${ }^{13}$ se refiere. Parte de los problemas de nuestras batallas socio-genéticas- tanto en el ámbito académico como en los entramados sociopolíticos macroscópicos que le cobijan- tienen que ver con el inexorable hecho avasallador de haber aceptado los términos de la conversación impuestos por las redes y el concierto de cánones hegemónicos que se entablan entre sí. Por ello, aunque conscientes de que el mero empleo de la categoría no implica la incorporación de sus valencias, como acto afirmativo estamos sustituyendo las voces, América Latina, Hispanoamérica, entre otras, con el mismo radical eurocéntrico. Preferiremos por mucho, la musicalísima Abya Yala.

Lo mismo puede señalarse en torno al pensamiento sobre la dimensión educativa. Paulatinamente, nuestras investigaciones han ido develando el mapa conceptual, y estamos en posición de afirmar que uno de los terrenos en donde puede observarse con nitidez la colonialidad del saber y su vocación de arrope es en la educación general. Al estudiar los años cincuenta en la FEG, la unanimidad en la reverencia pasmosa ante el canon, el binarismo maniqueo (polaridades excluyentes que privilegian la contraposición entre el "bien" y el "mal") de la guerra fría elevado a propuestas teóricas y académicas, la reificación de las grandes obras, la occidentosis curricular y la experimentación posible, siempre dentro de la "caja", revelan- a nuestro modo de ver- el despliegue de una contundente cartografía de colonialidad.

Tenemos en nuestro horizonte de trabajo la posibilidad de reconocer y estudiar la importancia de epistemologías otras (caribeñas, africanas,

11 Sosa op cit p. 148

12 Como hemos esbozado, estaremos apalabrando la voz, Abya Yala- el concepto testimonio del pueblo Kuna en la hoy Panamá- para referirnos a Latinoamérica. No obstante a que algunos académicos ya habían utilizado la expresión ABYA YALA en contraposición a la designación consagrada de América, la primera vez que la expresión fue explícitamente usada con sentido político fue en la Il Cumbre Continental de los Pueblos y Nacionalidades Indígenas de ABYA YALA, realizada en Quito en 2004. En rigor, fue en la III Cumbre Continental de los Pueblos y Nacionalidades Indígenas de ABYA YALA, realizada en Guatemala cuando por vez primera se convocan como ABYA YALA. También resuelven constituir una Coordinación Continental de las Nacionalidades y Pueblos Indígenas de ABYA YALA "como espacio permanente de enlace e intercambio, donde converjan experiencias y propuestas, para que juntos enfrentemos las políticas de globalización neoliberal y luchar por la liberación definitiva de nuestros pueblos hermanos, de la madre tierra, del territorio, del agua y de todo patrimonio natural para vivir bien". Paulatinamente, en los diferentes encuentros del movimiento de los pueblos originarios, el nombre América va siendo sustituido por ABYA YALA, "indicando así no apenas otro nombre, sino también la presencia de otro sujeto enunciador del discurso, hasta aquí callado y subalternizado en términos políticos: los pueblos originarios”. Información derivada de: Cronicasinmal.blogspot, en: metiendoruido.com/recuperado el 12 de enero de 2017. Hoy día, el concepto, Abya Yala denota una convicción y un posicionamiento decolonial hacia el buen vivir y otros paradigmas de transformación social y epistémica.

13 El concepto se ha empleado en la corriente que vincula a Piaget con Vigosky y Freire, es decir, en las regiones del pensamiento constructivista y la pedagogía de la liberación. (Hatano 1993). Más recientemente, Manuel Arias Maldonado, enunciando desde el paradigma del antropoceno, utiliza el concepto en el sentido de la explosión tecnológica y mediática en sus múltiples repercusiones de comunicación social. "Si tenía sentido hablar de un diálogo entre los medios tradicionales y sus consumidores, ahora nos encontramos con un "poliálogo" donde las conversaciones se entrecruzan y solapan, pero también discurren por caminos paralelos sin converger jamás”. Ver Internet contra la democracia, En Eurozine , (5 de octubre 2017) 
andinas), lo que nos permitirá advertir y alejarnos del entrampamiento que producen las "verdades universales" abriéndose la posibilidad de concebir también una educación general fundada en la pluralidad epistémica, en las interconexiones y en los contextos en los que se produce el saber y se llevan a cabo nuestras prácticas. El reto ha sido sacudido en nuestro contexto puertorriqueño por el colega Waldemiro Vélez (2019) cuando insiste en:

la importancia de incorporar ideas, voces y sentires de intelectuales del Caribe para abrir brechas hacia la decolonización del poder, del saber y del ser. Partiendo del convencimiento de que las aportaciones de ellos y ellas le añadirán nuevas energías a las Ciencias Sociales al interior de los Estudios Generales, enriqueciendo y ampliando los contextos en los que éstos se ubican y desde un lugar de enunciación otro que cuestiona las premisas eurocéntricas y universalistas -muy frecuentes en los Estudios Generales ayer y hoy- que han afirmado que lo mejor que se ha pensado y escrito proviene de Europa. Desde El Caribe las ciencias sociales y el mundo se entienden de otras maneras, por lo que rescatar esas ideas y ubicarlas en el contexto de los Estudios Generales podría ser una aportación interesante para el desarrollo de unos Estudios Generas situados y más capaces de propiciar el desarrollo de unas identidades latinoamericanas y caribeñas en los estudiantes Universitarios. (2019, p.84.) $)^{14}$

El problema de la dependencia intelectual de occidente, sobre todo de una construcción histórica y epistemológica de ese mundo, había sido cuestionada por autores como José Martí en 1891, José Carlos Mariátegui en 1928, Rodolfo Kusch en 1953 y Reinaga, en el 1970. ${ }^{15}$ Estos pensadores seminales habían sentado las bases teóricas para lo que actualmente se conoce como pensamiento decolonial. ${ }^{16}$ Incluso la "nave del olvido" rondaba muy cerca y a pesar de la contemporaneidad con varias de las fuentes del pensamiento decolonial de la década de los cincuenta, - Fanon, Cessaire, entre otros- la continuidad de la hegemonía del canon occidental, a través de la colonialidad triple del ser, el saber y el poder, hizo pasar totalmente desapercibida tales contribuciones para la intelectualidad puertorriqueña de la época. (Vélez, 2019).

Los años cincuenta marcaron un compás inexorable sobre un pentagrama inamovible: el binarismo ampuloso, hegemónico y devorador de todo tipo de conocimiento y, por ende, educación que no provenga del torrente mesiánico e infalible de la razón instrumental, los valores de occidente y el telos de un "progreso" con definición puramente capitalista. El movimiento de la educación general se produjo sobre este ciclorama de "guerra fría" y de un "lavado cerebral" intenso, que amenazaba en Puerto Rico, con otras faenas desmanteladoras. Su historia crítica requiere develar las circunstancias políticas en donde se desenvolvió; la comprensión de los alcances y limitaciones de las fórmulas concretas educativas de la FEG debe pasar por estas consideraciones.

En efecto, nuestro acercamiento a estos procesos y tensiones en Puerto Rico y fundamentalmente en los ámbitos de la educación universitaria, ha recibido gran influencia del pensamiento decolonial, lo que nos ha permitido contar con un prisma y fundamentos metodológicos que no han sido frecuentes en el abordaje de la historia de nuestro país. Al ubicar las posturas intelectuales de la época más allá del "truismo"17 que los puertorriqueños somos occidentales, y de que la puertorriqueñidad es meramente una forma de ser occidental, pudimos identificar lo que para muchos fue un agrio debate -algunos del lado del gobernador Muñoz Marín y otros del rector Benítez- como una zona de coincidencia en la que no se ponían en

Decolonizar las ciencias sociales para el desarrollo de unos Estudios Generales latinoamericanos y caribeños (2019) en Umbral Num 15, UPR ISSN 2518386.

15 Martí era cubano, Mariátegui, peruano, Kusch, argentino y Reinaga, boliviano, lo que atestigua lo presente que estaba esa reflexión en nuestro continente.

16 Ver particularmente el prólogo de una edición del libro de Mariátegui escrita por Aníbal Quijano (1979), principal precursor del pensamiento decolonial.

17 El concepto es un neologismo utilizado por Antonio J. Colorado y viene como anillo al dedo a este referente semiótico. 'Truismo' degenera de true, o verdad en inglés; una verdad incuestionable, algo que nadie pone en duda. 
duda los dogmas eurocéntricos que han guiado la mayor parte de nuestra historiografía.

\section{¿Colonialidad o colonialismo curricular en la} Facultad de Estudios Generales?

Existen ciertas premisas centrales por medio de las que enfocamos nuestro planteamiento sobre ambas categorías teóricas: el colonialismo y la colonialidad curricular en la FEG. En Puerto Rico, por la persistente relación de sujeción jurídico / político/militar hablamos de colonialismo en sentido clásico. Siendo conscientes de que el término colonialidad se utiliza para describir las maneras en las que se mantienen, y aún se fortalecen lazos de dependencia y control intelectual en países que han superado el colonialismo jurídico-político, lo que no es el caso de Puerto Rico, nos parece que las elaboraciones teóricas que se han venido desarrollando desde el pensamiento decolonial, son de extraordinaria ayuda para escudriñar y entender la situación de Puerto Rico, objeto de esta reflexión.

En efecto, lo que queremos plantear es que en Puerto Rico se produce un problema de doble pespunte. Es decir, una especie de colonialidad, que se imbrica con el colonialismo jurídico/ político/militar. Así, nos parece, que a partir de las herramientas teóricas y epistemológicas que nos provee el pensamiento decolonial podemos acercarnos críticamente a esta variante compleja que acontece en Puerto Rico y su academia. Se trata del colonialismo educativo y curricular estudiado con el lente decolonial.

Por ejemplo, y grosso modo, entra al proscenio la colonialidad, cuando aún en las fuerzas contestatarias y de resistencia, así como en las instituciones como la Universidad que reclaman y aspiran autonomías políticas y administrativas, se perfila el arrope del eurocentrismo y la occidentalidad.
En primera instancia y como fundamento, podemos aseverar el predominio holístico y unívoco del canon occidentalista y la ausencia del estudio de la realidad social, histórica y cultural de Puerto Rico, el Caribe y Abya Yala. Esto se revela diáfanamente en la filosofía educativa de la 'Educación General' en la Facultad de Estudios Generales y el carácter de su currículo'. La aspiración de extender los cursos medulares a dos años, por ejemplo, al modo de 'arrope', especialmente en los cursos de Humanidades y Ciencias Sociales con fuerte aroma occidentósico en sus propuestas de cursos es evidencia de lo anterior.

Por otro costado, he detectado una profunda 'megalofilia intelectual' en el contexto educativo en pro del universalismo/perennialismo. Nos referimos al modelo importado de la Universidad de Chicago de trabajar con 'Grandes obras', 'Grandes conferencias', v.g. Hay factores concurrentes y propiciantes al colonialismo curricular en la FEG, a saber: a) los profesores visitantes en la Facultad de Estudios Generales ${ }^{18}$, la intromisión curricular del militarismo norteamericano; el programa 'Átomos para la paz' y lo que he denominado, los embelesos co-curriculares entre los que anoto, los viajes de Estudios a Europa con su difusión tan avasallante, los 'Saludos inaugurales del rector' ${ }^{19}$ con su carga axiológica tan persuasiva, peculiar y carismática y el papel de las 'Fundaciones filantrópicas,' entre otros escenarios den inmersión.

Canon occidentalista y ausencia de la realidad social, histórica y cultural puertorriqueña en la FEG de Puerto Rico

Veamos en algún detalle el predominio del canon occidentalista y la ausencia de la realidad social, histórica y cultural de Puerto Rico en la Facultad de los Estudios Generales. En rigor, esta arista recoge el sabor de todas las demás. Durante la década del cincuenta del siglo XX el occidentalismo no era puesto en duda, ni como paradigma intelectual ni como uno de los elementos identitarios principales de los puertorriqueños. Ya fuera al interior o al

18 Por 'profesor Visitante' debemos entender una categoría de reclutamiento per se en el régimen de personal de la UPR. No son profesores que meramente visitan Puerto Rico y la Universidad por razones diversas. Todavía en la reglamentación vigente, la Sección 120.32 de; Reglamento General de la UPR define los Profesores Visitantes cómo Personas de gran prestigio, reconocidas por la comunidad académica, que se invitan a dictar cursos o seminarios con crédito y que normalmente se contratan por un período académico. El asunto de los 'profesores visitantes' tuvo múltiples dimensiones y por ende resulta irreductible a generalizaciones elementales en este momento. 
exterior de la Universidad de Puerto Rico, el canon occidental permanecía incuestionable. La Facultad de Estudios Generales, lejos de ser la excepción a este patrón generalizado, fungió como uno de sus principales baluartes.

Cabe destacar que desde sus inicios en los años cuarenta, el emblemático curso de Humanidades llevaba por título: "Introducción a las Civilizaciones de Occidente" y en su descripción se observaba la importancia del conocimiento de esa cultura¡como si fuera homogénea! - para la formación del estudiante universitario. ${ }^{20}$ Muy pronto, en 1947, este curso se extendió a dos años, constituyéndose en un caso revelador y evidente de expansión en pro de una dirección curricular determinada. Empero, la tendencia es rastreable en todos los cursos de la FEG y en el Programa de Bachillerato (licenciatura en la nomenclatura de América Latina) con el modelo de "Grandes Obras" que se implantó en muchos de sus ofrecimientos.

El imaginario esparcido por el rector Jaime Benítez, entre otros, era palmario: lo más excelso que se ha pensado en cualquier tiempo y lugar, se ha pensado en Occidente. No se trata ingenuamente de aseverar que no existían culturas no occidentales, sino que la principal responsabilidad de la educación general estribaba en poner en contacto a los estudiantes universitarios con el concepto de 'excelencia', y de ninguna manera con la diversidad de culturas y aportaciones intelectuales que existen en el mundo. En la medida en que el occidentalismo estaba tan fuertemente arraigado en nuestra semiótica e idiosincrasia intelectual, no se entendía necesario siquiera explicitar los criterios o razones para la exclusión de otras culturas y formas de pensar y de ser. Basta con su procedencia distinta a lo occidental para su descualificación, ya que las únicas ideas que ostentaban validez universal (en cualquier tiempo y lugar) eran las occidentales. ${ }^{21}$ Es precisamente por lo anterior que se entendía que los mejores materiales educativos para los estudios generales consistían en las "Grandes Obras de la Cultura Occidental".
La pregunta fundamental que tendríamos que abordar es: ¿Qué consecuencias tuvo y tiene todavía este entendido en la filosofía educativa y en el plan de estudios (currículo) de la Facultad de Estudios Generales? Aunque la respuesta a esta pregunta merece una ponderación muy cuidadosa, somos del parecer que el exagerado énfasis en informar o fundamentar, tanto la filosofía educativa como el programa de estudios de la FEG en el canon occidental y eurocéntrico, ha sido y probablemente sigue siendo empobrecedor y limitante para el desarrollo de la FEG y de los esfuerzos por una educación integral y significativa. Después de cerca de setenta y cinco años, aún el curso de humanidades continúa centrado en la cultura occidental y se aventura muy poco -cuando lo hace- en el estudio y reconocimiento de las aportaciones intelectuales de culturas que anteceden o trascienden a occidente.

Uno de los principales problemas que se nos presenta es que la exclusión de culturas con las que nos hemos relacionado y, de alguna manera, formado en Puerto Rico, como la proveniente de las epistemes africanas, por ejemplo, nos ha dificultado el desarrollo de una identidad más inclusiva y diversa como universitarios y puertorriqueños. Además, el afecto desmedido a "verdades" de validez universal desemboca en el rezago con respecto a tendencias de gran trascendencia en la ciencia y la filosofía de los siglos XX y XXI.

Nuestro compromiso es aportar para que el canon occidental se entienda como una forma, entre otras, de acercarnos al conocimiento y a la educación, válida por supuesto, pero de ninguna manera excluyente. Es preciso señalar que, aunque el canon occidental no era directamente cuestionado desde la FEG, sí se desarrollaba un proceso en el que no pocos de los docentes adscritos a esta unidad impulsaban la necesidad de incorporar la situación prevaleciente en Puerto Rico, tanto como contexto a la totalidad de los ofrecimientos curriculares como una materia en sí misma (un curso sobre Puerto

20 Maldonado Rivera, Vélez Cardona y Sánchez Zambrana (2013), pp. 111-112.

21 Algunos indicios de lo que eventualmente fue concebido por autores como Said y Boaventura como orientalismo, puede rastrearse en la trayectoria curricular de la FEG, en particular con algunas exploraciones en el Departamento de Humanidades. Resulta interesante, por ejemplo, el tema del ensayo de George Atiyeh sobre el Eluma Elish babilónico en sus similitudes con la teogonía de Hesíodo y el génesis hebreo. Ver REG Vol. 1 Num 1 Dic 1958 De este autor conocemos un trabajo pionero sobre las epistemes no-occidentales. Los clásicos orientales en la educación liberal. Ver REG Vol.2 Num. 3, (1958) pp. 2 y 20. 
Rico). Esto, dado el entendido, y probablemente por las influencias que la obra de John Dewey había tenido tanto en la Universidad de Chicago como en toda la educación superior norteamericana, de que es a partir de su propia situación existencial que- de modo idóneo- podrán los estudiantes conectar e incorporar los nuevos conocimientos.

También, estaba sobre el tapete la insatisfacción con la situación política de Puerto Rico, considerada por diversos actores de la época como francamente colonial. Enese contexto, lanegación o invisibilización del país en los ofrecimientos educativos de la Universidad -hemos apuntado con anterioridadpodría catalogarse como una estrategia política que buscaba "despuertorriqueñizar al puertorriqueño" (Marqués, 1956). Nos parece que estamos ante una situación mucho más compleja que lo que a simple vista podría proyectarse, ya que el insistente reclamo por la incorporación de la realidad puertorriqueña, más que limitarse a una postura 'insularista' o una especie de complejo provincial, podría significar en el contexto de Puerto Rico particularmente, un planteamiento categórico en contra el universalismo eurocéntrico y en contra de la asimilación al ámbito cultural y económico de los Estados Unidos.

A pesar de ciertos reclamos, unos más tímidos y otros más contundentes, sobre la importancia de incorporar la realidad puertorriqueña como parte importante de los ofrecimientos de la FEG, ellos no recibieron la consideración debida. Esto aun cuando en algún momento provinieron del propio Consejo Superior de Enseñanza. Las razones son múltiples y sumamente complejas dada la situación política por la que atravesaba y aún atraviesa Puerto Rico. Algunas de ellas las examina con rigor el colega Waldemiro Vélez en sus líneas de investigación relacionadas a las tensiones entre el gobernador Muñoz Marín y el rector Jaime Benítez, sus repercusiones en la FEG, en la UPR y en todo Puerto Rico (Sánchez Zambrana et al. (2019). ${ }^{22}$

\section{Conclusiones}

La Facultad de Estudios Generales, tal y como se decantó con buen torrente en las esferas académicas del Puerto Rico de los años cincuenta del siglo XX adoptó, se curtió, asimiló y persiguió las expresiones polares del movimiento de la Educación General en las universidades norteamericanas o la genuflexión acrítica a la gran elocuencia de los conferenciantes españoles y latinoamericanos solo podía retrasar esta sociogénesis filosófica y curricular.

Por consiguiente, y habida cuenta del cuadro que se pigmentaba en la vida cultural de nuestra joven FEG, consideramos que había un rumbo luminoso entre sus posibilidades. Era en la integración del conocimiento en donde atisbamos que la ruta identitaria requeriría de una filosofía educativa consecuente y de unos desarrollos curriculares articulados. Empero, la integración que se necesitaba y que atisbamos como producente, consistía en una de carácter complejo, transdisciplinar y decolonial.

El contexto político y politizado arrollaba en favor de los ideales de la modernidad, del éxito económico como el telos del progreso, del american way of life, el "My Way" de Sinatra. Y fue tan avasallador en los años cincuenta que no permitió el asomo siquiera de ese enfoque integrativo. Y ha permanecido así, constreñido como un bonsái, como una "Ceiba en un tiesto".

¿Es la Universidad de Puerto Rico, a través de su enfoque de los estudios generales, un caso aislado en Abya Yala, en cuanto a colonialismo curricular se refiere? Si así fuera, la dosis de provincialismo que permea este estudio sobre la Facultad de Estudios Generales de la Universidad de Puerto Rico obnubilaría sus posibilidades educativas. De todas formas, presentamos una apuesta por la metodología heurística que empleamos, misma que nos ha llevado a develar un reservorio de documentos que no alcanzaron la estatura de advenir a publicaciones y que, por ende, pasan por debajo del radar del investigador (Memoriales, actas, minutas, correspondencias v.g.). Sin embargo, estoy convencido de que- aparte de esta alusión metodológica existen algunos aprendizajes y reflexiones específicas de esta 'pequeña gran historia' de la FEG, susceptibles de extrapolar para el examen critico de las instituciones de Educación 
Superior en el Caribe y Abya Yala. Cabe la pregunta: ¿Cómo otras instituciones pueden mirarse a través de esta reflexión?

Buscando que esta deliberación pueda obtener otros alcances, adelanto algunos elementos a modo de propuesta, ante el escenario planteado de doble sujeción: colonialismo y colonialidad curricular.

Aspiramos en los Estudios Generales a la elaboración paulatina y robusta de un currículo basado en tres pilares: a) la Integración del conocimiento, para evadir la parcelación y atomización de los planos de la realidad; b) la transdisciplinariedad para sumar las voces excluidas de la academia, a la par con que asume, procesa y transgrede las parcelas del saber; y c) la decolonialidad, para zafarnos del arrope epistémico occidentósico que penetra las entrañas de la academia y se instala en las 'venas abiertas' de los pueblos bajo la hegemonía del canon del sistema mundo. Un currículo tal se provee como arma educativa de crecimiento, concienciación y búsqueda de una sociogénesis identitaria, crítica, auténtica y orgánica para nuestros pueblos y naciones. Como paso concreto, en la Red Internacional de Estudios Generales (RIDEG) se están labrando esfuerzos en pro de una Maestría Abya Yala en Estudios Generales, (MAYEG) de currículo decolonial y transdisciplinario y a base de un formato inter-universitario e itinerante.

Vuelta a la semilla de este escorzo: resulta frecuente escuchar de parte de exalumnos y la comunidad referidos de elogio hacia los cursos de primer año de la Facultad de Estudios Generales del Recinto de Río Piedras de la Universidad de Puerto Rico: ¡Qué importantes fueron esos cursos! -exclaman.

En muchos sentidos, somos depositarios de un afecto heredado por aquellos, que, dentro de ese tupido laberinto de inoculaciones y neblinas, pudieron mantener la simiente o la tea de una criticidad permanente, digamos, un tanto latente, pero disponible en reservorio para brotar y encender ante estímulos adecuados venideros. Tal es la esperanza de estos tiempos en donde la 'salsa estival' de la canícula borincana se impone de maneras misteriosas y sonoras, como en el verano 2019. Y en donde, al calor la singularidad pandémica, podremos exclamar que "a pesar del otoño" o del colonialismo curricular: jcreceremos!"

\section{Referencias bibliográficas}

Azócar, R. E. (28 de febrero de 2012). Policrisis. Aporrea.

Borsani, M. E. (2014). Reconstrucciones metodológicas y/o metodologías a posteriori. Astrolabio, (13). pp. 146-168.

Colorado, A. (1955). Puerto Rico y la cultura occidental mss. En AFLMM, Sección V, Gobernador de Puerto Rico. Documentos misceláneos, sub serie \# 7 .

Espinosa Gómez, D. R. (2014). Una escuela sentipensante: Reflexiones hacia una escuela diferente. Autores-editores, (2018).

Haber, A. (2011). Nometodología Payanesa: Notas de metodología indisciplinada (con comentarios de Henry Tantalean, Francisco Gil García y Dante Angelo). Revista Chilena de Antropología, (23). https:// doi.org/10.5354/0719-1472.2011.15564

Maldonado Rivera, M., Vélez Cardona, W. y Sánchez Zambrana, C. (2013). Historia crítica de la Educación General en Puerto Rico. Ediciones Mágica.

Maldonado-Torres, N. (2007). "Sobre la colonialidad del ser: contribuciones al desarrollo de un concepto" en Santiago Castro-Gómez y Ramón Grosfoguel (comps.). El giro decolonial. Reflexiones para una diversidad epistémica más allá del capitalismo global. Bogotá, Universidad JaverianaInstituto Pensar, Universidad Central- iesco, Siglo del Hombre Editores.

Marqués, R. (1956, 1971). Prólogo a Juan Bobo y la dama de occidente: Pantomima puertorriqueña para un ballet occidental. Ed. Cultural, San Juan.

Mignolo, W. (2010). Desobediencia epistémica. Retórica de la modernidad, lógica de la colonialidad y gramática de la descolonialidad. Ediciones del Signo. pp. 126.

Rinaldi, S. (enero-junio, 2019a). La relación currículum-género en carreras universitarias de Ciencia Política en Argentina. Revista de Educación Superior en América Latina, (5), 26-30. https://doi.org/10.14482/esal.5.378.82 
Rinaldi, S. (2019b). Perspectiva nordeurocéntrica en el currículum universitario de ciencia política [artículo en línea]. Revista Contribuciones a las Ciencias Sociales. https:// www.eumed.net/rev/cccss/2019/03/curriculum-ciencia-politica.html

Sánchez Zambrana, C. J., Vélez Cardona, W. y Maldonado Rivera, M. (2019). La Educación General en Puerto Rico: La década de los cincuenta. Ediciones Mágica.

Sosa Elízaga, R. (2005). Herencias y retos del conocimiento en América Latina. Sociologías, (14), 130-148. https://doi.org/10.1590/S1517$\underline{45222005000200007}$
Vélez Cardona, W. (2019). Decolonizar las ciencias sociales para el desarrollo de unos Estudios Generales latinoamericanos y caribeños (2019) en Revista Umbral Núm. 15, UPR ISSN 251-8386

Walsh, C., Schiwy, F. y Castro-Gómez, S. (2002). Indisciplinar las ciencias sociales: Geopolíticas del conocimiento y colonialidad del poder: Perspectivas desde lo andino. Universidad Andina Simón Bolívar y Abya-Yala. 